\title{
Tabakproduktegesetz: Ein Umdenken ist erforderlich
}

\section{Theodor Abelin}

Prof. Dr. med., MPH, ehem. Direktor des Instituts für Sozial- und Präventivmedizin der Universität Bern, ehem. Mitglied des WHO Expert Advisory Panel on Tobacco or Health (1977-1997) und der Eidg. Kommission für Tabakprävention (1999-2007), Präsident (1973-1992), Vorstandsmitglied (1992-2010) der Arbeitsgemeinschaft Tabakprävention, Mitglied der FMH

Die bisherige Geschichte des Tabakproduktegesetzes zeigt, wie wenig im Parlament - und wohl auch in einer breiteren Öffentlichkeit - das Ausmass der gesundheitlichen Folgen des Tabakkonsums wahrgenommen wird. Es geht nicht um gelegentliche gesundheitliche Nebenerscheinungen einer in der Bevölkerung traditionell verankerten Gewohnheit, sondern darum, ob das Parlament weiterhin mit einem Wirtschaftszweig zusammenarbeiten soll, dessen Produkt bei einer Mehrzahl seiner Kunden zu Abhängigkeit und bei der Hälfte zu tödlichen Krankheiten führt. Die Ärzteschaft ist sich der Problematik bewusst, und dem praktizierenden Arzt bieten sich Möglichkeiten, in und ausserhalb der Praxis zur Meinungsbildung beizutragen. In den folgenden Ausführungen geht es darum, auf

\section{Résumé}

La loi sur les produits du tabac doit être reformulée, ce qui nécessite un changement de paradigme. Le modèle commercial de l'industrie du tabac s'appuie sur une dépendance souvent insurmontable à la nicotine, qui apparaît lors d'une consommation ordinaire et enfreint ainsi le droit humain fondamental à l'intégrité physique. Cela vaut également pour les nouveaux produits à base de nicotine sans tabac, dont l'impact à long terme ne sera pas connu avant quelques décennies. Afin d'instaurer des mesures strictes contre ces activités, une avocate néerlandaise prépare, avec le soutien public déclaré de cliniques de cancérologie et de sociétés de discipline médicale, un procès pénal à l'encontre de multinationales du tabac. Des préparatifs analogues sont en cours dans d'autres pays.

De ce point de vue, il faut une loi sur les produits du tabac qui limite la vente à des magasins agréés et aux seules personnes dépendantes, comme dans le cas des médicaments sur ordonnance ou des substances toxiques.

Afin de responsabiliser les décideurs politiques, l'implication personnelle de tous les médecins engagés est nécessaire, vis-à-vis des patients et amis ainsi qu'au sein des organisations professionnelles de médecins. Ces dernières peuvent, souvent en tant que membres des associations professionnelles cantonales, par exemple se distancer publiquement du rôle de l'Union suisse des arts et métiers en tant que porte-parole des intérêts de l'industrie du tabac. Et ce d'autant plus que les PME doivent assumer une part disproportionnée des coûts indirects du tabagisme à hauteur de plusieurs milliards de francs. einige der grundlegenden Fragen einzugehen, um die es bei dieser Meinungsbildung geht, und einige Möglichkeiten aufzuzeigen, die sich der Ärzteschaft in diesem Zusammenhang anbieten.

\section{Nikotinabhängigkeit als Krankheit}

Als Ärzte wissen wir, dass die Schwierigkeit, mit Rauchen aufzuhören, weitgehend auf das durch die Tabakpflanze produzierte Nikotin zurückzuführen ist, dessen Konsum bei einem grossen Teil der Konsumenten in kurzer Zeit zu zerebralen Änderungen im Sinne einer Zunahme von Nikotinrezeptoren und schwerer Abhängigkeit führt. Die internationale Klassifikation der Krankheiten anerkennt diese als Krankheit (ICDCode F17) [1]. Damit ist die Nikotinabhängigkeit - auch unabhängig von den körperlichen Folgen des Rauchens - als Krankheit zu betrachten.

\section{Das Geschäftsmodell der Nikotin- industrie}

Die Tabakindustrie hat sich diesen Ablauf zunutze gemacht und darauf gestützt ein raffiniertes und für sie äusserst lukratives Geschäftsmodell entwickelt, das kurz zusammengefasst so aussieht:

"Man macht Neukonsumenten nikotinsüchtig und kann ihnen in der Folge über Jahrzehnte ihre tägliche Dosis Nikotin verkaufen. Scheinbar an Erwachsene gerichtete Werbung hat eigentlich Jugendliche im Visier. Indem man vorgibt, ein Produkt nur für Erwachsene zu vertreiben, macht man Kinder neugierig und verführt Jugendliche zum Konsumversuch. Die Zahl der Nikotinrezeptoren im Hirn vermehrt sich - die Abhängigkeit ist erreicht, und der zukünftige Absatz ist gesichert» [2].

\section{Beeinträchtigung der körperlichen Unversehrtheit}

In anderen Worten nimmt das Geschäftsmodell der Tabakindustrie - die eigentlich «Nikotinindustrie» genannt werden sollte - in Kauf, dass sie ein Produkt ver- 
treibt, welches bei der beabsichtigten Art der Benützung bei einem grossen Teil der Konsumenten und Konsumentinnen durch die Entwicklung der Nikotinabhängigkeit die körperliche Unversehrtheit beeinträchtigt. Es gibt kein Lebensmittel mit vergleichbaren Auswirkungen bei einem Konsum in den beabsichtigten Mengen: Weder die gesundheitlich problematischen Süssgetränke noch fettreiche oder andere im Übermass die Gesundheit schädigende Lebensmittel führen bei einem Konsum in der vorgesehenen Menge zu Abhängigkeit und/oder gesundheitlichen Störungen. Deshalb war es im Rahmen der letzten Revision des Lebensmittelgesetzes folgerichtig, Tabakprodukte aus diesem Gesetz zu entfernen und mit einem eigenen Gesetz - dem Tabakproduktegesetz - zu regeln ebenso wie dies auch für andere Produkte mit einem Gefährdungspotential der Fall ist (Arzneimittelgesetz, Chemikalien- bzw. früher Giftgesetz). Diese Produkte dürfen nur durch dafür lizenzierte Stellen beziehungsweise an als berechtigt ausgewiesene Abnehmer abgegeben werden.

\section{Es gibt kein Lebensmittel mit vergleichbaren Auswirkungen bei einem Konsum in den beabsichtigten Mengen.}

Bei Zigaretten, aber auch bei Zigarren, Stumpen und Pfeife kommt dazu, dass als Vehikel für das Nikotin Tabakrauch verwendet wird, mit den für diese Produkte bekannten zusätzlichen gesundheitlichen Folgen. Es sei nur an die fast 20-fache Risikoerhöhung für Lungenkrebs und eine solche um 100\% für Herzinfarkt bei Rauchern von 20 oder mehr Zigaretten pro Tag erinnert, um deutlich zu machen, welche Gesundheitsbeeinträchtigung beim Verkauf dieser Produkte in Kauf genommen wird. Es ist daher unerlässlich, die Marktzulassung aller Nikotinprodukte restriktiv zu regeln und die Werbung und öffentliche Promotion wie etwa bei den rezeptpflichtigen Medikamenten gänzlich zu verbieten.

\section{Unklare Schädlichkeit der neuen Nikotin- produkte}

Besondere Aufmerksamkeit erfordert der Umgang mit den neuen Nikotinprodukten wie E-Zigaretten, bei denen das auf Nikotin gestützte Geschäftsmodell der Tabakfirmen ebenfalls zum Tragen kommt. Bemerkenswert ist, dass die Tabakkonzerne bei der Promotion dieser Produkte betonen, sie seien weniger schädlich als die bisher üblichen Zigaretten - womit sie die Beeinträchtigung der körperlichen Unversehrtheit durch Zigaretten unzweideutig eingestehen. Der Ver- kauf von Zigaretten beruht heute also nicht auf einer behaupteten Annahme der Unschädlichkeit, sondern erfolgt im vollen Bewusstsein der damit bewirkten körperlichen Beeinträchtigung.

Was die neuen Nikotinprodukte betrifft, bedeutet «weniger schädlich» nicht notwendigerweise «unschädlich». Denn angesichts der enormen Schädlichkeit des Zigarettenrauchs wäre auch "nur» eine Risikoverdoppelung für Lungenkrebs oder eine Risikoerhöhung um «nur» 20 Prozent für Herzinfarkt noch immer inakzeptabel. Um solche Risikoerhöhungen nachzuweisen, sind aufgrund der langen Latenzzeiten auf Jahrzehnte angelegte Langzeitstudien erforderlich. Man erinnere sich nur: Als Anfang der 1950er Jahre in England und den USA die ersten Fall-Kontrollstudien über Rauchen und Lungenkrebs publiziert wurden, waren seit den 1920er Jahren, in denen das Rauchen weit verbreitet wurde, drei Jahrzehnte vergangen, während deren sich Karzinome und kardiale Störungen entwickeln konnten. Und noch in den 1950er Jahren waren die Schäden des Rauchens auch unter Medizinern nicht allgemein anerkannt. Als der jetzige Autor 1958 als cand.med. anlässlich einer Autopsie eines an Lungenkrebs Verstorbenen den Professor fragte, ob es stimme, dass Lungenkrebs mit Rauchen zu tun habe, erhielt er zur Antwort: «Stellen Sie keine dummen Fragen.» Erst in den 1960er Jahren standen die ersten Longitudinalstudien zur Verfügung und ermöglichten dem amerikanischen «Surgeon General», die Schädlichkeit des Rauchens in ihrem ganzen Ausmass offiziell zu bestätigen [3]. Die neuen Nikotinträger heute als unschädlich oder sogar als therapeutisch nützlich frei zuzulassen, wie es die Nikotinindustrie und ihre Verbündeten fordern, wäre angesichts der langen Latenzzeiten bis zur Feststellung einer allfälligen langfristigen Gesundheitsstörung unverantwortlich.

\section{Strafrechtliche Verfolgung wegen syste- matischer bewusster Körperverletzung mit möglichen tödlichen Folgen}

Das Recht auf körperliche Unversehrtheit ist in verschiedenen Verfassungen und internationalen Vertragswerken als Menschenrecht garantiert, und auch in Art. 10 Abs. 2 der schweizerischen Bundesverfassung ist dieses Prinzip verankert. Wie ist es dann möglich, dass die Tabak- oder Nikotinindustrie ungestraft ein Produkt vertreiben darf, von dem feststeht, dass es bei beabsichtigtem Gebrauch zu Abhängigkeit und bei rund der Hälfte der Konsumenten zu Krankheit und Tod führt?

Diese Frage stellt sich auch die niederländische Anwältin Bénédicte L. M. Ficq, und sie beabsichtigt nun, im 
Namen von an Lungenkrebs erkrankten Patienten, die jahrelang erfolglos versucht haben, sich von ihrer Nikotinabhängigkeit zu befreien, eine strafrechtliche Verfolgung der Tabakindustrie einzuleiten wegen systematischer bewusster Körperverletzung mit möglichen tödlichen Folgen. Dabei wird sie, wie am niederländischen Fernsehen mitgeteilt wurde, von mehreren

Insoweit als sich Ärztegesellschaften nicht öffentlich von der Tabakpolitik ihres Gewerbeverbandes distanziert haben, haben sie diese stillschweigend mitunterstützt.

namhaften Krebskliniken und Ärzteorganisationen unterstützt. Schon in den 1990er Jahren war in den Vereinigten Staaten mit einer ähnlichen Begründung zivilrechtlich gegen die Tabakindustrie vorgegangen worden, und die damalige Verurteilung der Tabakkonzerne zu einem Schadenersatz von 6 Milliarden Dollar zur Benützung in der Tabakprävention zeigt, dass der Sachverhalt der Verletzung der körperlichen Unversehrtheit im Zusammenhang mit dem Nikotinabusus und dessen Folgen durchaus mit Erfolg gerichtlich geahndet werden kann. Die niederländische Anwältin steht bereits auch mit deutschen und norwegischen Organisationen im Präventionsbereich in Verbindung, so dass auch dort und in weiteren Staaten entsprechende Prozesse erwartet werden können [4].

\section{Was bedeutet das für das Vernehmlas- sungsverfahren zum Tabakprodukte- gesetz?}

Diese Überlegungen bedeuten, dass es nicht nur moralisch, sondern auch staats- und strafrechtlich fragwürdig ist, auf ein Tabakproduktegesetz hinzuarbeiten, das im Widerspruch zu dem in der Verfassung garantierten Menschenrecht auf körperliche Unversehrtheit steht. Zwar muss anerkannt werden, dass ein beträchtlicher Teil unserer Bevölkerung nikotinabhängig ist und Zugang zu entsprechenden Produkten haben sollte. Doch wurde die Entfernung von Tabakprodukten aus dem Lebensmittelgesetz nicht vorgenommen, um einfach mit den alten Regelungen fortzufahren, auch wenn dies die Tabaklobby mit ihren Verbündeten verlangt. Ebenso wie bei rezeptpflichtigen Medikamenten oder Giftstoffen ist eine Formel erforderlich, bei der Zigaretten und ähnliche Nikotinprodukte gezielt nur durch berechtigte Abgabestellen und nur an Abhängige abgegeben werden - auch wenn die Nikotinwirtschaft dem Konsumenten zu suggerieren versucht, sein Verlangen nach entsprechenden Produkten sei frei gewählt und diene zu seinem Vergnügen. Und ebenso wie die Publikumswerbung für rezeptpflichtige Medikamente stark eingeschränkt oder verboten ist, sollte dies auch für nikotinhaltige Produkte gelten. Als Nicht-Jurist kann ich nicht beurteilen, ob sich auch Parlamentarier und Parlamentarierinnen schuldig machen, wenn sie entgegen besserem Wissen ein Gesetz erlassen, das eine Verletzung der körperlichen Integrität eines grossen Teils der Bevölkerung zulässt.

\section{Was ist dabei die Rolle des Arztes und seiner Berufsorganisationen?}

Um bei Parlamentariern in diesem Zusammenhang das Verantwortungsbewusstsein zu fördern, genügen gelegentliche allgemein gehaltene Aufrufe von breit abgestützten Dachorganisationen nicht. Denn Bürger und Politiker werden wenig beeindruckt sein, wenn der Aufruf von einer einzigen oder von wenigen Organisationen kommt, die die Tabakprävention bereits in ihrem Namen tragen. Doch wenn nacheinander Aufrufe von Seiten verschiedener ärztlicher Kantonalund Bezirksvereine sowie spezialärztlicher Organisationen bekannt gemacht werden, so wird deren Botschaft grössere Chancen haben, in der Bevölkerung und bei den Parlamentariern das erforderliche Problembewusstsein zu fördern. Und um dies zu organisieren, wird es nicht ohne Elemente einer strategischen Planung gehen.

\section{Wer soll wann die Initiative ergreifen?}

Initiativen kommen letztendlich von motivierten und beherzten Individuen, und so kann es hier nicht primär darum gehen, Ärzte- oder Gesundheitsorganisationen kollektiv zum Handeln aufzufordern. Was jedoch möglich sein sollte, ist, engagierte Leser und Leserinnen zu ermutigen, selbst Initiativen zu ergreifen - sei es im Gespräch mit Patienten oder Freunden, deren Engagement im gesellschaftlichen und politischen Prozess eine Rolle spielen könnte, oder sei es im Rahmen ihrer Standes- und Berufsorganisationen, die der Bevölkerung und der Politik zeigen können, wie wichtig es für sie ist, dass in der Schweiz das Menschenrecht auf körperliche Unversehrtheit ernst genommen wird.

\section{Arztpraxen, KMUs und der Gewerbe- verband}

Im Rahmen der bisherigen parlamentarischen $\mathrm{Be}$ handlung des Tabakproduktegesetzes ist der Schweizerische Gewerbeverband als vehementer Wortführer der Tabakinteressen aufgetreten. Mehrere kantonale Ärztegesellschaften sind Mitglied des Gewerbeverban- 
des ihres Kantons, und insoweit als sie sich nicht öffentlich von der Tabakpolitik ihres Gewerbeverbandes distanziert haben, haben sie diese stillschweigend mitunterstützt.

Dabei gäbe es gute Gründe für unsere Ärztegesellschaften, sich im Rahmen ihres Gewerbeverbands nicht nur von dessen tabakfreundlicher Politik zu distanzieren, sondern ihn auch zu überzeugen, dass eine aktive Mitarbeit in der Tabakprävention im direkten wirtschaftlichen Interesse der von ihm vertretenen kleinen und mittelgrossen Unternehmen (KMUs) läge. Der Grund liegt in den indirekten gesellschaftlichen Kosten des Rauchens, die für die Schweiz auf jährlich rund 3,9 Milliarden Franken geschätzt worden sind [5] und kleinere und mittlere Betriebe besonders hart treffen. Denn längere krankheitsbedingte oder durch Tod verursachte und noch nicht ersetzte Ausfälle von Mitarbeitenden können in solchen Betrieben häufig nicht wie in Grossbetrieben durch Aufteilung der Aufgaben auf mehrere gesunde Mitarbeiter kompensiert werden, und so treffen diese direkt den Geschäftsgang. KMUs sind deshalb gerade auch bei tabakbedingten Krankheitsausfällen besonders stark betroffen und haben einen entsprechend überdurchschnittlichen Teil der indirekten wirtschaftlichen Kosten des Rauchens zu tragen. Umso nützlicher wäre eine wirksame Prävention im Nikotinbereich für die Mitglieder des Schweizerischen Gewerbeverbands.

Bereits Vorstösse einzelner engagierter Ärzte und Ärztinnen könnten genügen, um innerhalb der kantonalen Ärztegesellschaften zu entsprechenden Diskussionen zu führen und im besten Fall innerhalb des Gewerbeverbandes eine Bewegung zugunsten eines präventiv wirklich wirksamen Tabakproduktegesetzes auszulösen.

\section{Literatur}

1 ICD International Statistical Classification of Diseases and Related Health Problems. 10th Revision (ICD-10). World Health Organization WHO, Geneva, 2016.

2 Abelin T. Nikotin-, nicht nur Tabakprävention. Der Bund, Bern, 18. Januar 2018.

3 Smoking and Health. Report of the Advisory Committee to the Surgeon General of the Public Health Service. Public Health Service Publication No. 1103, U.S. Department of Health, Education, and Welfare, Washington, DC, 1964.

4 Ficq BLM, et al. Klageschrift. Deutsche Übersetzung, 2016: https:// sickofsmoking.nl/wp-content/uploads/2017/02/Dutch-CriminalComplaint-Tobacco-Industry-German.pdf

5 Fueglister-Dousse S, Jeanrenaud C, Kohler D, Marti J. Kosten und Nutzen von Präventionsmassnahmen. Kurzfassung, Institut de recherches économiques, Université de Neuchâtel, 2009. 